\title{
A 2D integrable axion model and Target space duality
}

\author{
Péter Forgács \\ Laboratoire de Mathématiques et Physique Théorique \\ CNRS UMR 6083 \\ Université de Tours \\ Parc de Grandmont, 37200 Tours, France
}

\begin{abstract}
A review is given on the recently proposed two dimensional axion model $(\mathrm{O}(3) \sigma$-model with a dynamical $\theta$-term) and the $\mathrm{T}$-duality relating it to the $\mathrm{SU}(2) \times \mathrm{U}(1)$ symmetric anisotropic $\sigma$-model. Strong evidence is presented for the correctness of the proposed $S$-matrix for both models comparing perturbative and Thermodynamical Bethe Ansatz calculations for different types of free energies. This also provides a very stringent test of the validity of T-duality transformation at the quantum level. The quantum non-integrability of the $\mathrm{O}(3) \sigma$-model with a constant $\theta$-term, in contradistinction to the axion model, is illustrated by calculating the $2 \rightarrow 3$ particle production amplitude to lowest order in $\theta$.
\end{abstract}




\section{The axion model}

Let us consider the following two dimensional $\sigma$-model defined by the Lagrangian円:

$$
\mathcal{L}=\frac{1}{2 \tilde{\lambda}} \partial_{\mu} n^{a} \partial^{\mu} n^{a}+\frac{\tilde{\lambda}}{32 \pi^{2}(1+\tilde{g})} \partial_{\mu} \theta \partial^{\mu} \theta+\frac{\theta}{8 \pi} \epsilon^{\mu \nu} \epsilon^{a b c} n^{a} \partial_{\mu} n^{b} \partial_{\nu} n^{c}
$$

where $\tilde{\lambda}, \tilde{g}$ are (real) parameters (couplings) and the $n$-fields are subject to the constraint $n^{a} n^{a}=1$. I shall refer to (1.1) as the 'axion model' since it is the $\mathrm{O}(3)$ nonlinear $\sigma$-model with a dynamical $\theta$-term, which is in turn a natural two-dimensional analogue of its phenomenologically important four-dimensional counterpart, in the same way as the $\mathrm{O}(3) \sigma$-model is considered to be a $2 \mathrm{D}$ analogue of $4 \mathrm{D}$ non-abelian gauge theories. As will be exhibited later (1.1) is asymptotically free for the coupling range $-1<\tilde{g} \leq 0$. The theory defined by (1.1) is an $\mathrm{O}(3)$ non-linear $\sigma$-model, coupled to a scalar field, $\theta$, (whose normalization has been chosen for later convenience) through the Hopf term. The latter is proportional to the topological current of the $\mathrm{O}(3)$ model and with the normalization chosen in (1.1) its space-time integral (after a Wick rotation) yields the topological charge, which is integer valued (for non singular configurations). The variable $\theta$ therefore has a natural interpretation as an angle, $\theta \in[0,2 \pi]$, so that the three dimensional target space of the axion model is topologically $S^{2} \times S^{1}$. Although at the classical level only one of the parameters (couplings), $(\tilde{\lambda}, \tilde{g})$, is relevant I prefer to introduce them from the very beginning as in the quantum theory both couplings play a rôle. It is not difficult to see that for $\tilde{g} \rightarrow-1$ the $\theta$ field decouples from $n^{a}$, i.e. one obtains the $\mathrm{O}(3) \sigma$-model and a decoupled free scalar field. There is another special value of the coupling, $\tilde{g}=0$, when (1.1) turns out to be canonically equivalent to the $\mathrm{O}(4) \sigma$-model.

The 'manifest' global symmetries of the axion model are $\mathrm{SO}(3) \times \mathrm{SO}(2)_{\theta}$, where $\mathrm{SO}(2)_{\theta}$ generates a shift $\theta \rightarrow \theta+$ const., a symmetry of (1.1) up to a total derivative.

In this paper I shall review the axion model and its dual, the $\mathrm{SU}(2) \times \mathrm{U}(1)$ symmetric anisotropic principal $\sigma$-model. The most remarkable feature of the axion model is its conjectured quantum integrability (in the sense that there is no particle production). It allows one to deduce by bootstrap methods its exact spectrum, which turns out to depend on the value of the (renormalization group invariant) coupling ratio $\tilde{p}=2 \pi(1+\tilde{g}) / \tilde{\lambda}$. As long as $0<\tilde{p}<1$ the spectrum consists of $\mathrm{SO}(3)$ triplets and singlets ('breathers') as well as of doublets ('kinks'), while for $\tilde{p}>1$ only two doublets.

This review is organized in the following way: In Section 2 it is shown that the classical equations of motion of the axion model admit a (standard) Lax pair,

\footnotetext{
${ }^{1}$ The following conventions are used: for a vector $v$ in two-dimensional Minkowski space $v^{\mu} v_{\mu}=v_{+} v_{-}$where $v_{ \pm}=v_{0} \pm v_{1}$. The antisymmetric tensor is defined by $\epsilon^{01}=1, \tau^{a}=\sigma^{a} / 2$ with $\sigma^{a}$ being the standard Pauli-matrices satisfying $\sigma^{a} \sigma^{b}=\delta^{a b}+i \epsilon^{a b c} \sigma^{c}, a, b, c=1,2,3$ and summation is implied over the repeated indices.
} 
implying integrability (in the sense that an infinite set conserved quantities exists). Next in Subsection 2.2 the axion model is demonstrated to be equivalent to the rather well studied $\mathrm{SU}(2) \times \mathrm{U}(1)$ symmetric anisotropic $\sigma$-model by a simple target-space duality transformation. This observation leads to a new Lax pair.

Section 3 is devoted to perturbative investigations of the two models. First a brief renormalization group analysis of the anisotropic model is carried out, emphasizing the important property of asymptotic freedom. Then the two loop $\beta$-functions are compared in the two models with the conclusion that they are equivalent provided one takes into account a change of the renormalization scheme. In Subsections 3.2, and 3.3 the Legendre transformation of various (bosonic and fermionic type) finite density ground state energies (free energies) is computed in the anisotropic model. In Subsection 3.4 the main points of the analogous computation in the axion model are discussed. It is found that the free energies fully agree in perturbation theory. Since one compares physical quantities in the two (dual) models, no scheme dependence arises and therefore this provides good evidence for the validity of a quantum version of this T-duality.

The anisotropic $\sigma$-model is generally believed to be quantum integrable. Applying the thermodynamical Bethe Ansatz one can also compute the free energies nonperturbatively from the bootstrap $S$-matrix and the comparison with the perturbative results yields quite a stringent test of the proposed $S$-matrix. This program is carried out in Section 4.

First in Subsection 4.1, in the framework of the form factor bootstrap approach, the $2 \rightarrow 3$ particle production amplitude in the $\mathrm{O}(3) \sigma$-model with a constant $\theta$-term is shown to be non vanishing. To the best of my knowledge this is the first quantitative evidence for the (generally expected) quantum non-integrability of this model. To my mind this fact underlines the rather surprising nature of the quantum integrability of the axion model. Subsection 4.2 is devoted to the thermodynamical Bethe Ansatz and in 4.3 the exact relation between the infrared and the ultraviolet mass scales $(m / \Lambda$-ratio) is given. Finally in Subsection 4.4 the limit $\tilde{p} \rightarrow 0(\mathrm{O}(3)$ limit $)$ is discussed, in particular it is explained how one recovers the known $m / \Lambda$-ratio of the $\mathrm{O}(3) \sigma$-model.

\section{Lax pairs and T duality}

\subsection{A Lax pair for the axion model}

The axion model belongs to a family of $\mathrm{O}(3)$ symmetric classically integrable $\sigma$-models discovered in Ref. [1], some of whose results I now recall. Introducing the matrix valued current

$$
I_{\mu}=\frac{\tilde{\lambda}}{8 \pi} n \epsilon_{\mu \nu} \partial^{\nu} \theta-\frac{\sqrt{\tilde{g}}}{2} \epsilon_{\mu \nu} \partial^{\nu} n+\frac{1}{2} n \partial_{\mu} n
$$


where $n=i n^{a} \sigma^{a}$, the equations of motion of (1.1) can be written as:

$$
\partial^{\mu} I_{\mu}=0, \quad \partial_{\mu} I_{\nu}-\partial_{\nu} I_{\mu}=\left[I_{\mu}, I_{\nu}\right]
$$

The standard form (2.2) of the equations of motion allows for the introduction of a Lax pair,

$$
U_{ \pm}=\frac{1}{1 \pm \omega} I_{ \pm}
$$

satisfying the zero curvature equation

$$
\partial_{\mu} U_{\nu}-\partial_{\nu} U_{\mu}=\left[U_{\mu}, U_{\nu}\right]
$$

for all values of the spectral parameter $\omega$. The current, $I_{\mu}$, is closely related to the matrix valued Noether current, $\mathcal{N}_{\mu}=-i \tau^{a} \mathcal{N}_{\mu}^{a}$, defined by $\delta \mathcal{L}=\partial^{\mu} \varepsilon^{a} \mathcal{N}_{\mu}^{a}$ corresponding to the symmetry transformation $\delta n^{a}=\epsilon^{a b c} \varepsilon^{b} n^{c}$ :

$$
I_{\mu}=\tilde{\lambda} \mathcal{N}_{\mu}+\epsilon_{\mu \nu} \partial^{\nu} T, \quad T=\left(\frac{\tilde{\lambda}}{8 \pi} \theta-\frac{\sqrt{\tilde{g}}}{2}\right) n .
$$

Note that the trivially conserved part of $I_{\mu}\left(\epsilon_{\mu \nu} \partial^{\nu} T\right)$ is essential for the zero curvature equation (2.4) to be satisfied.

\subsection{T-dual of the axion model}

Here I show that the axion model (1.1) is classically equivalent to a rather well studied $\sigma$-model, the so-called anisotropic $\mathrm{SU}(2)$ principal $\sigma$-model (PCM). The equivalence is a canonical one given by an Abelian target space (T-)duality transformation [2, 3]).

The Lagrangian of the anisotropic PCM can be written as:

$$
\mathcal{L}_{\Sigma}=-\frac{1}{2 \lambda}\left\{L_{\mu}^{a} L^{a \mu}+g L_{\mu}^{3} L^{3 \mu}\right\}
$$

where

$$
L_{\mu}=G^{-1} \partial_{\mu} G=\tau^{a} L_{\mu}^{a},
$$

and $g$ is the parameter of anisotropy. The Lagrangian (2.6) can be interpreted as a deformation of the $\mathrm{SU}(2) \times \mathrm{SU}(2)$ (or $\mathrm{O}(4)$ ) symmetric nonlinear $\sigma$-model by the parameter $g$. For a generic value of $g$ this (torsionless) model has an $\mathrm{SU}(2)_{\mathrm{L}} \times \mathrm{U}(1)_{\mathrm{R}}$ symmetry. At the classical level the anisotropic model, (2.6), interpolates between the $\mathrm{SU}(2) \times \mathrm{SU}(2)(g=0)$ and $\mathrm{O}(3)(g=-1)$ models.

The equations of motion of the anisotropic principal model (2.6) can be written entirely in terms of the current $L_{\mu}$ :

$$
\partial^{\mu} L_{\mu}^{3}=0, \quad \partial^{\mu} L_{\mu}^{1}=-i g L^{2 \mu} L_{\mu}^{3}, \quad \partial^{\mu} L_{\mu}^{2}=i g L^{1 \mu} L_{\mu}^{3} .
$$


It is known that the equations of motion (2.8) admit a Lax representation [1, (5], i.e. there is a spectral parameter dependent current, $V_{\mu}=\tau^{a} V_{\mu}^{a}$, satisfying the zero curvature equation (2.4). This current can be written as:

$$
V_{ \pm}^{1,2}=\alpha_{ \pm} L_{ \pm}^{1,2}, \quad V_{ \pm}^{3}=a_{ \pm} L_{ \pm}^{3}
$$

where

$$
\alpha_{ \pm}=-\frac{4+g \omega^{2}}{4-g \omega^{2} \pm 4 \omega}, \quad a_{ \pm}=-\frac{4-g \omega^{2} \mp 4 g \omega}{4-g \omega^{2} \pm 4 \omega} .
$$

To exhibit the classical T-duality transformation mapping the axion model (1.1) to the anisotropic PCM (2.6) [6], it is convenient to parametrize the $n^{a}$-fields in Eq. (1.1) as

$$
n^{1}=\sin \vartheta \sin \varphi, \quad n^{2}=\sin \vartheta \cos \varphi, \quad n^{3}=\cos \vartheta, \quad \theta=-\frac{4 \pi}{\tilde{\lambda}} \sqrt{1+\tilde{g}} \chi,
$$

in terms of which the Lagrangian of the axion model (1.1) (after an integration by parts) becomes

$$
\mathcal{L}=\frac{1}{2 \tilde{\lambda}}\left\{\partial_{\mu} \vartheta \partial^{\mu} \vartheta+\sin ^{2} \vartheta \partial_{\mu} \varphi \partial^{\mu} \varphi+\partial_{\mu} \chi \partial^{\mu} \chi+2 \sqrt{1+\tilde{g}} \cos \vartheta \epsilon^{\mu \nu} \partial_{\mu} \chi \partial_{\nu} \varphi\right\} .
$$

An Abelian T-duality transformation [2] with respect to the $\mathrm{SO}(2)_{\theta}$ symmetry of the axion model (1.1), corresponds to the following canonical transformation [3]:

$$
\chi^{\prime}=-\frac{\tilde{\lambda}}{\sqrt{1+\tilde{g}}} p_{\alpha} \quad p_{\chi}=-\frac{\sqrt{1+\tilde{g}}}{\tilde{\lambda}} \alpha^{\prime},
$$

where (and in the following) $p_{\chi}$ resp. $p_{\alpha}$ denote the canonical momenta conjugate to $\chi$ resp. to its 'dual' $\alpha$. In terms of the new variable, $\alpha$, the dual Lagrangian turns out to be:

$\mathcal{L}_{\Sigma}=\frac{1}{2 \tilde{\lambda}}\left\{\partial_{\mu} \vartheta \partial^{\mu} \vartheta+\left(1+\tilde{g} \cos ^{2} \vartheta\right) \partial_{\mu} \varphi \partial^{\mu} \varphi+(1+\tilde{g})\left[\partial_{\mu} \alpha \partial^{\mu} \alpha+2 \cos \vartheta \partial_{\mu} \alpha \partial^{\mu} \varphi\right]\right\}$.

A simple calculation shows that (2.14) is nothing but the Lagrangian (2.6), when parametrizing the $\mathrm{SU}(2)$ valued field, $G$, by the Euler angles

$$
G=e^{i \varphi \tau^{3}} e^{i \vartheta \tau^{1}} e^{i \alpha \tau^{3}}
$$

and taking into account the relations at the classical level between the couplings:

$$
\tilde{\lambda}=\lambda, \quad \tilde{g}=g .
$$

The observation that the axionic model is the $\mathrm{T}$ dual of $\mathcal{L}_{\Sigma}$ also explains why $\theta$ should be interpreted as an angular variable. Indeed, as it has been shown in 
Ref. [7] the Abelian T duality transformation (2.13) maps the target space of the isotropic PCM, $S^{3}$, the Abelian T duality (2.13) into $S^{2} \times S^{1}$. The arguments of Ref. [7] can be easily adopted to the present case with $g>-1$, and it is clear that in Eq. (1.1) $n^{a}$ parametrize an $S^{2}$ and $\theta$ parametrizes an $S^{1}$.

One can now use the classical T-duality transformation (2.13) to map the linear system of the axion model (2.2) to a new Lax pair for the anisotropic $\sigma$ model (2.6). It is given by Eq. (2.3), where the current, $I_{\mu}$, has to be replaced by

$$
\hat{I}_{\mu}=\partial_{\mu} G G^{-1}+g\left(G \tau^{3} G^{-1}\right) L_{\mu}^{3}-i \sqrt{g} \epsilon_{\mu \nu} \partial^{\nu}\left(G \tau^{3} G^{-1}\right) .
$$

Eq. (2.17) is obtained from (2.1) by the T-duality transformation (2.13). $\hat{I}_{\mu}$ is related to the Noether current $\hat{\mathcal{N}}_{\mu}$, corresponding to the manifest symmetry $\delta G=-i \varepsilon^{a} \tau^{a} G$ of $(2.6)$ and can be written analogously to $\mathcal{N}_{\mu}$ :

$$
\hat{I}_{\mu}=\lambda \hat{\mathcal{N}}_{\mu}+\epsilon_{\mu \nu} \partial^{\nu} \hat{T}, \quad \hat{T}=-i \sqrt{g} G \tau^{3} G^{-1} .
$$

It is clear that the new Lax pair (2.3) and the 'old' one, (2.9), cannot be related by a gauge transformation since they have different pole structures as functions of the spectral variable, $\omega$. In the $\tilde{g} \rightarrow-1$ limit, the axion model reduces to the original $\mathrm{O}(3) \sigma$-model (decoupled from the $\theta$ field), and the Lax pair (2.3) becomes equivalent to that of Ref. [B], where it has been pointed out that the corresponding $\hat{I}_{\mu}$ 's are ultralocal currents. I remark that the Lax pairs (2.3) and (2.9) correspond to (different) deformations of the usual Lax pairs of the principal chiral $\sigma$-model, linear in $\partial_{\mu} G G^{-1}$ respectively $G^{-1} \partial_{\mu} G$.

\section{Quantum equivalence}

\subsection{Perturbative investigations}

The canonical equivalence between the axion and the anisotropic PCM models naturally raises the question if there is also a quantum equivalence between them. Or putting it a slightly different way, one can ask if there is a quantum version of T-duality transformation (2.13).

The equivalence of the models in perturbation theory (PT) is certainly a necessary condition for the validity of quantum T-duality. I start by recalling some renormalization properties of the anisotropic $\sigma$-model and the important property of asymptotic freedom. Then I present a quantitative comparison in PT between the two models by comparing their $\beta$-functions.

Due to its $\mathrm{SU}(2)_{\mathrm{L}} \times \mathrm{U}(1)_{\mathrm{R}}$ symmetry, the anisotropic $\sigma$-model (2.6) is renormalizable in perturbation theory. (Of course, in Eq. 2.6) $(\lambda, g)$ should be interpreted as the bare couplings $\left(\lambda_{0}, g_{0}\right)$.) Using dimensional regularization, the relation between the bare parameters, $\left(\lambda_{0}, g_{0}\right)$, and the renormalized ones, $(\lambda, g)$, 
is written as:

$$
\lambda_{0}=\mu^{\epsilon} \lambda Z_{\lambda}, \quad \text { and } \quad 1+g_{0}=(1+g) Z_{g} .
$$

In Eq. (3.1) the dimensional parameter, $\mu$, is introduced as usual, to carry the mass dimension of $\lambda_{0}$ (which is dimensionless in 2D). The renormalization constants contain only simple poles in $\epsilon=2-n$ whose residues can be calculated in perturbation theory.

Physical quantities depend on the renormalized couplings $\lambda, g$ and the dimensional parameter, $\mu$, in such a way that the action of the renormalization group (RG) operator

$$
\mathcal{D}=\mu \frac{\partial}{\partial \mu}+\beta_{\lambda}(\lambda, g) \frac{\partial}{\partial \lambda}+\beta_{g}(\lambda, g) \frac{\partial}{\partial g},
$$

vanishes on them, where the $\beta$-functions are obtained from the residues of the first order poles. The RG equations for the running couplings $\bar{\lambda}, \bar{g}$ can be written as

$$
\begin{array}{ll}
\frac{d \bar{\lambda}}{d t}=\beta_{\lambda}(\bar{\lambda}, \bar{g}), & \bar{\lambda}(0)=\lambda, \\
\frac{d \bar{g}}{d t}=\beta_{g}(\bar{\lambda}, \bar{g}), & \bar{g}(0)=g .
\end{array}
$$

One is interested in the 'large time' asymptotic behaviour of the couplings as functions of an external field (or chemical potential) $h=h_{0} e^{t}$, with $h_{0}$ fixed and $t \rightarrow \infty$. In the anisotropic PCM the $\beta$-functions are known to be of the form:

$$
\begin{aligned}
& \beta_{\lambda}=-\frac{\lambda^{2}}{4 \pi}\left\{1-g+\lambda p_{2}(g)+\cdots\right\}, \\
& \beta_{g}=\frac{\lambda g(1+g)}{2 \pi}\left\{1+\lambda q_{1}(g)+\cdots\right\},
\end{aligned}
$$

where the two-loop $\beta$-function coefficients are:

$$
p_{2}(g)=\frac{1-2 g+5 g^{2}}{8 \pi}, \quad \text { and } \quad q_{1}(g)=\frac{1-g}{4 \pi} .
$$

Asymptotically free (AF) behaviour occurs when $\bar{\lambda} \rightarrow 0$ together with $\bar{g} \rightarrow g_{1}$ as $t \rightarrow \infty$, where $g_{1}$ is some constant. Analyzing Eqs. (3.4), (3.5), one finds three different AF solutions:

$$
\begin{aligned}
& \text { 1. } g_{1}=0 \quad g \equiv g_{0} \equiv \bar{g} \equiv 0, \\
& \text { 2. } g_{1}=-1 \quad g \equiv g_{0} \equiv \bar{g} \equiv-1 \text {, } \\
& \text { 3. } g_{1}=-1 \quad-1<\bar{g}<0 \text {. }
\end{aligned}
$$

Solutions (3.6) resp. (3.7) correspond to the $\mathrm{O}(4)$ resp. the $\mathrm{O}(3) \sigma$-model, while the solution (3.8) corresponds to the 'generic' case of the anisotropic PCM. 


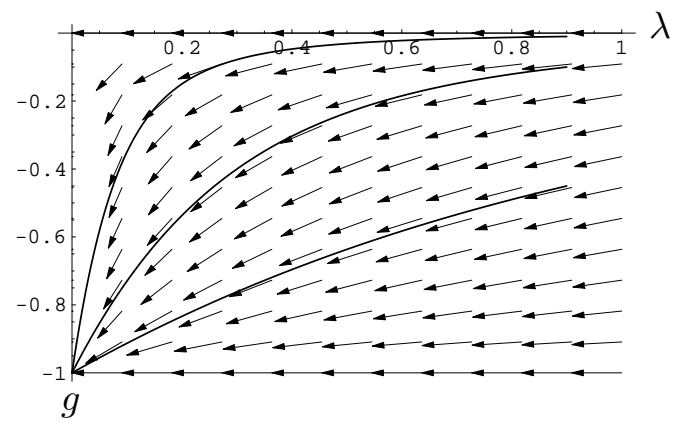

Figure 1: 1 loop flow diagram of the couplings $(\lambda, g)$

It is very convenient to introduce an exact $R G$ invariant combination of the two couplings

$$
p=2 \pi \lim _{t \rightarrow \infty} \frac{1+g(t)}{\lambda(t)},
$$

and an effective $\beta$-function for $\lambda(t)$ by

$$
\beta_{\text {eff }}(\lambda, p)=\beta_{\lambda}(\lambda, \Gamma(\lambda, p)),
$$

expressing $g(t)$, in terms of the running coupling, $\lambda(t)$, and the RG invariant quantity $p$ as $g(t)=\Gamma(\lambda(t), p)$. Using the perturbative result for $\Gamma(\lambda(t), p)$ [9] one finds

$$
\beta_{\mathrm{eff}}(\lambda, p)=\beta_{\mathrm{eff}}(\tilde{\lambda}, \tilde{p})=-\frac{\lambda^{2}}{2 \pi}+\frac{p-2}{8 \pi^{2}} \lambda^{3}+\cdots .
$$

Eq. (3.11) allows for the introduction of an RG-invariant $\Lambda$-parameter in the $\overline{\mathrm{MS}}$ scheme in the usual way:

$$
\Lambda_{\overline{\mathrm{MS}}}=\mu e^{-\frac{2 \pi}{\lambda}}\left(\frac{\lambda}{2 \pi}\right)^{\left(\frac{p}{2}-1\right)} e^{\gamma}\{1+\mathcal{O}(\lambda)\}
$$

The effective coupling $\lambda_{\text {eff }}(h)$ is defined by the transcendental equation

$$
\frac{2 \pi}{\lambda_{\mathrm{eff}}}+\left(\frac{p}{2}-1\right) \ln \frac{2 \pi}{\lambda_{\mathrm{eff}}}=\ln \frac{h}{\Lambda_{\overline{\mathrm{MS}}}},
$$

which is a function of the 'physical' parameter

$$
s=\ln \frac{h}{\Lambda_{\overline{\mathrm{MS}}}},
$$

only. Moreover the running coupling can be expressed in terms of $\lambda_{\text {eff }}(h)$ perturbatively (in the sense that it is an infinite power series). The asymptotic expansion (for large $s$ ) of the effective coupling (containing terms $\propto \ln s$ ) can be written as

$$
\lambda_{\mathrm{eff}}=\frac{2 \pi}{s}+\frac{\pi(p-2)}{s^{2}} \ln s+\cdots .
$$


After this detailed discussion of the renormalization properties of the anisotropic PCM model I just briefly recall the main point for the axion model. As discussed in detail in Ref. [6] the axion model is also renormalizable in the field theoretical sense and at one loop order the $\beta$-functions of the couplings of the axion model, $\beta_{\tilde{\lambda}}, \beta_{\tilde{g}}$, are simply obtained from $\beta_{\lambda}, \beta_{g}$ by the classical relation (2.16). At two loops, however, it has been found in [6] when using the background field method and dimensional regularization that the following perturbative redefinition of the couplings

$$
\tilde{\lambda}=\lambda+\frac{\lambda^{2}}{4 \pi}(1+g), \quad \tilde{g}=g+\frac{\lambda}{4 \pi}(1+g)^{2},
$$

(i.e. a change of scheme) is induced by the T-duality transformation. Taking into account Eqs. (3.16) the two loop $\beta$-functions of the two models turn out to be equivalent. Alternatively, defining the renormalization group invariant combination of the two couplings analogously as in the PCM model Eq. (3.9):

$$
\tilde{p}=2 \pi \lim _{t \rightarrow \infty} \frac{1+\tilde{g}(t)}{\tilde{\lambda}(t)},
$$

one finds

$$
p=\tilde{p}
$$

up to two loops [6]. Thus as far as coupling constant renormalization is concerned, the two models are equivalent, both are asymptotically free, and the actual value of $p$ effects only the two loop coefficient.

\subsection{Perturbative tests of free energies}

Up to now quantum equivalence between the two dually related models has been tested by comparing simple renormalization properties and the (two loop) $\beta$ functions. The fact that the higher coefficients of the $\beta$-functions are scheme dependent makes such a comparison more difficult and less conclusive. To lend some more credibility to the proposed perturbative quantum equivalence between the two models it is clearly desirable to compare physical quantities. For this purpose it is quite convenient to compute various zero temperature free energies obtained by minimizing the Legendre transform of the Hamiltonian density coupled to some conserved currents

$$
\hat{\mathcal{H}}=\mathcal{H}_{0}-h_{i} J_{0}^{i}, \quad \hat{H}=\int d x \hat{\mathcal{H}}=H-h_{i} Q_{i}
$$

where $h_{i}$ are constant external fields having the interpretation of chemical potentials. In the limit of large fields $\left(h_{i} \rightarrow \infty\right)$ corresponding to considering the system at large density, due to asymptotic freedom weak coupling perturbation theory (in $\lambda$ resp. $\tilde{\lambda}$ ) is applicable. 
The Legendre transform of the ground state energy density of the system must be of the form:

$$
\mathcal{F}(h) \equiv \delta f(h) \equiv f(h)-f(0)=-h^{2} F_{0}(h / \Lambda, Q)
$$

where $\Lambda$ is a parameter of dimension mass. (Eq. (3.19) follows simply from dimensional analysis.) In an asymptotically free theory the result of a perturbative computation of $F_{0}$ (which is of course renormalization group invariant) is an asymptotic series in the running coupling, $\bar{\lambda}(h / \Lambda) \propto[\ln (h / \Lambda)]^{-1}$, where $\Lambda$ is the usual renormalization group invariant combination of the (arbitrary) mass scale, $\mu$, and the renormalized coupling, $\lambda$.

Since $\mathcal{F}(h)$ is a physical quantity, the results obtained in the two models should agree. Thus comparing them provides a further nontrivial check on the quantum equivalence between the axion and the anisotropic $\sigma$-model, hence also on the validity of the T-duality transformation at the quantum level.

Since the axion field, $\theta$, is actually an angle, its winding number (topological charge) can be non trivial. Therefore I present here the Legendre transformation of the modified Hamiltonian (3.18) for a rather general case including also topological charges.

Let us consider a general sigma model with torsion

$$
\mathcal{L}_{0}=\frac{1}{2} g_{A B} \partial^{\mu} X^{A} \partial_{\mu} X^{B}+\frac{1}{2} b_{A B} \epsilon^{\mu \nu} \partial_{\mu} X^{A} \partial_{\nu} X^{B}
$$

and the following Ansatz for a set of conserved currents

$$
J_{\mu}^{i}=\mathcal{C}_{A}^{i}(X) \partial_{\mu} X^{A}+\epsilon_{\mu}{ }^{\nu} \mathcal{B}_{A}^{i}(X) \partial_{\nu} X^{A}
$$

sufficiently general to include topological currents. The Legendre transformation of (3.18) yields the Lagrangian of the modified model which can be written as

$$
\hat{\mathcal{L}}=\mathcal{L}_{0}+h^{i} J_{0}^{i}+\frac{1}{2} h^{i} h^{j} \mathcal{C}_{A}^{i} \mathcal{C}^{A j}
$$

In fact $\hat{\mathcal{L}}$ can be obtained by gauging $\mathcal{L}_{0}$ i.e. by the substitution

$$
\partial_{\mu} X^{A} \rightarrow \partial_{\mu} X^{A}+h^{i} \delta_{\mu 0} \mathcal{C}^{i A}
$$

when the antisymmetric field $b_{A B}$ is invariant (without compensating gauge transformation) under the symmetry transformation generated by the conserved currents (3.21).

\subsection{The anisotropic PCM model}

I recall next the main results of the perturbative computation of $\mathcal{F}$ in the anisotropic PCM (2.6) first (for more details see Ref. [9]). There are two conserved Noether 
charges in the model (2.6), $Q_{\mathrm{L}}, Q_{\mathrm{R}}$, corresponding to the $\mathrm{U}(1)_{\mathrm{L}} \times \mathrm{U}(1)_{\mathrm{R}}$ transformation

$$
\delta G=i \epsilon_{L} \sigma^{3} G-i \epsilon_{R} G \sigma^{3} .
$$

Introducing two chemical potentials coupled to the $Q_{\mathrm{L}}$ resp. $Q_{\mathrm{R}}$, charges the Hamiltonian (3.18) takes the form:

$$
H=H_{\Sigma}-h_{\mathrm{L}} Q_{\mathrm{L}}-h_{\mathrm{R}} Q_{\mathrm{R}}
$$

Since the anisotropic PCM is torsionless the Legendre transformation of (3.25) can be simply found using the gauging procedure (3.23). To actually calculate the ground state energy in PT one has to pass to the Euclidean field theory formulation in which case the chemical potentials $h_{i}$ can be interpreted as constant, imaginary gauge fields. The corresponding covariant derivative $D_{\mu}$ is then given by

$$
D_{2} G=\partial_{2} G+h_{L} \sigma^{3} G-h_{R} G \sigma^{3}, \quad D_{1} G=\partial_{1} G .
$$

The gauged Lagrangian can be written as:

$$
\mathcal{L}=\mathcal{L}_{0}+\mathcal{L}_{1}+\mathcal{L}_{2}
$$

where $\mathcal{L}_{1}$ and $\mathcal{L}_{2}$ denote the terms linear and quadratic in the external fields, respectively.

The computation of the free energy is based on the generating functional

$$
e^{-\int d^{n} x f(h)}=\int \mathcal{D} G \exp \left\{-\int d^{n} x\left(\mathcal{L}_{0}+\mathcal{L}_{1}+\mathcal{L}_{2}\right)\right\}
$$

In PT one expands Eq. (3.28) in powers of the (bare) coupling $\lambda_{0}$. I have also written the volume element as $d^{n} x$, where $n=2-\epsilon$ to indicate that dimensional regularization is employed in the perturbative calculations.

In perturbation theory the action has to be expanded around a (stable) solution of the classical equations of motion. This amounts to find elements of $\mathrm{SU}(2), G_{0}$, that correspond to (local) minima of the quadratic part of the gauged Lagrangian,

$$
\mathcal{L}_{2}=-\frac{2 h_{L}^{2}}{\lambda_{0}}\left(1+g_{0} z^{2}\right)-\frac{2 h_{R}^{2}\left(1+g_{0}\right)}{\lambda_{0}}+\frac{4 h_{L} h_{R}\left(1+g_{0}\right) z}{\lambda_{0}}
$$

where

$$
z=\frac{1}{2} \operatorname{Tr}\left\{\sigma^{3} G^{-1} \sigma^{3} G\right\} .
$$

The first solution, referred to as 'bosonic', (BOS) is given as:

$$
G_{0}=\frac{1}{\sqrt{2}}\left(1+i \sigma^{2}\right), \quad h_{R}=0,
$$


and the other one considered here, which I call 'fermionic' (FER) is

$$
G_{0}=i \sigma^{2} .
$$

(The reason for the names 'bosonic' resp. 'fermionic' will become clear later on.)

I start with the BOS case first. The leading, $\mathcal{O}\left(\lambda_{0}^{-1}\right)$, term in perturbation theory is given by the potential energy, $\mathcal{L}_{2}$, at its minimum, which is immediately seen to be $\left(z_{0}=0, h_{R}=0\right)$

$$
\mathcal{F}_{\text {BOS }}^{(-1)}=\mathcal{L}^{(-1)}=-\frac{2 h_{L}^{2}}{\lambda_{0}} .
$$

The 1-loop term leads to the following integral:

$$
\mathcal{F}_{\mathrm{BOS}}^{(0)}=\frac{4 h_{L}^{2}}{n} \int \frac{d^{n} p}{(2 \pi)^{n}} \frac{\left(1+g_{0}\right) p_{2}^{2}-g_{0} p^{2}}{\left(p^{2}\right)^{2}-4 g_{0} h_{L}^{2} p^{2}+4\left(1+g_{0}\right) h_{L}^{2} p_{2}^{2}} .
$$

For details concerning the actual computation, see Ref. [9], here I just quote the final $R G$ improved perturbative result for the asymptotic expansion of the free energy for large values of the external fields:

$$
\mathcal{F}_{\mathrm{BOS}}(h)=-\frac{h^{2}}{\pi}\left\{s+\left(1-\frac{p}{2}\right) \ln s+\left(\ln 2-\frac{1}{2}\right)+\cdots\right\} .
$$

In the FER case the leading (classical) term of the free energy is

$$
\mathcal{F}_{\mathrm{FER}}^{(-1)}=\mathcal{L}^{(-1)}=-\frac{2\left(1+g_{0}\right)}{\lambda_{0}}\left(h_{L}+h_{R}\right)^{2} .
$$

Note that $\mathcal{F}^{(-1)}$ for the diagonal charge depends only on the sum $h=h_{L}+h_{R}$. In the present case one needs to expand $\mathcal{F}(h)$ up to $\mathcal{O}\left(\lambda_{\text {eff }}^{2}\right)$ in the effective coupling, which would at first sight necessitate a three-loop computation. In fact one obtains all the $\mathcal{O}\left(\lambda_{\text {eff }}^{2}\right)$ terms from the one loop result alone! This 'mini miracle' is due to the $(1+g)^{2}$ factor in front of the one-loop term together with the fact that $(1+\bar{g})^{2}=\mathcal{O}\left(\lambda_{\text {eff }}^{2}\right)$, implying that the one-loop term is already $\mathcal{O}\left(\lambda_{\text {eff }}^{2}\right)$. The result turns out to be:

$$
\mathcal{F}_{\mathrm{FER}}(h)=-\frac{p h^{2}}{\pi}\left\{1-\frac{p}{4 \pi} \lambda_{\text {eff }}+\frac{p^{2}}{32 \pi^{2}} \lambda_{\text {eff }}^{2}+\frac{p \lambda_{\text {eff }}^{2}}{8 \pi^{2}}\left[\ln p+\ln \left(\frac{\lambda_{\text {eff }}}{2 \pi}\right)\right]+\mathcal{O}\left(\lambda_{\text {eff }}^{3}\right)\right\} .
$$

Note the non-analytic contribution, $\ln \lambda_{\text {eff }}$, in the last term of (3.37). The large $s$ expansion in the FER case is finally given as

$$
\mathcal{F}_{\mathrm{FER}}(h)=-\frac{p h^{2}}{\pi}\left\{1-\frac{p}{2 s}-\frac{p^{2}}{4 s^{2}} \ln s+\frac{p}{2 s^{2}}\left[\ln p+\frac{p}{4}\right]+\cdots\right\} .
$$

The above form of $\mathcal{F}(h)$ Eq. (3.37) explains the name 'fermionic' as its leading term corresponds to the free energy of $p$ free fermions. The $\mathcal{O}(1 / s)$ terms can 
be interpreted as interaction terms. In the 'bosonic' case the leading term in Eq. (3.35) is logarithmically divergent.

Finally note that the one loop term also depends only on the sum $h_{L}+h_{R}$, just like the classical one (3.36). This is not an accident. As shown in Ref. 9], in the FER case the free energy does depend only on the sum $h_{L}+h_{R}$ to all orders in perturbation theory. In fact it is essential that $\mathcal{F}\left(h_{L}, h_{R}\right)$ be a function of $h=h_{L}+h_{R}$ in order to be able to match the perturbative result with the corresponding one computed by the non-perturbative Thermodynamical Bethe Ansatz for the DIAG case in Subsection 4.2.

\subsection{The axion model}

Next I outline the computation of the corresponding ground state energies to one loop order in the axion model (1.1), starting with the BOS case first. With the Euler angle parametrization of $G$ (2.15) the $\mathrm{U}_{\mathrm{L}}(1)$ transformation, $G \mapsto$ $e^{i \kappa \tau^{3}} G$ of the anisotropic $\sigma$-model (2.6) acts as a simple shift, $\varphi(x) \mapsto \varphi(x)+$ $\kappa$. The corresponding Noether charge, $Q_{\mathrm{L}}$, and its image under the T-duality transformation, $\tilde{Q}_{\mathrm{L}}$, are simply

$$
Q_{\mathrm{L}}=\int d x p_{\varphi}, \quad \tilde{Q}_{\mathrm{L}}=\int d x \tilde{p}_{\varphi}, \quad \text { where } \quad p_{\varphi}=\frac{\partial \mathcal{L}_{\Sigma}}{\partial \dot{\varphi}}, \tilde{p}_{\varphi}=\frac{\partial \mathcal{L}}{\partial \dot{\varphi}}
$$

since the canonical transformation implementing the T-duality mapping (2.13) effects only $p_{\alpha}, \chi^{\prime}, \alpha^{\prime}$ and $p_{\chi}$, leaving the other fields, $\varphi, \vartheta, p_{\varphi}, p_{\vartheta}$, unchanged.

Since in the BOS case the $b_{A B}$ field in Eq. (1.1) is invariant, one can simply 'gauge' the Lagrangian of the axion model in an external $\left(h_{\mathrm{L}}\right)$ field (see Eq. (3.23). The classical ground state is found to be $\varphi \equiv \chi \equiv 0, \vartheta \equiv \pi / 2$. (The corresponding solution of the anisotropic $\sigma$-model is given by $\varphi \equiv \alpha \equiv 0, \vartheta \equiv \pi / 2$.)

Expanding the (Euclidean) Lagrangian (after suitable rescalings, etc.) one obtains

$$
\overline{\mathcal{L}}=-\frac{2 h_{\mathrm{L}}^{2}}{\tilde{\lambda}_{0}}+\frac{1}{2} m \mathcal{M} m^{T}+\mathrm{o}(\tilde{\lambda})
$$

where

$$
\mathcal{M}=\left(\begin{array}{ccc}
-\partial^{2}+4 h_{\mathrm{L}}^{2} & 0 & 2 h_{\mathrm{L}} \sqrt{1+\tilde{g}_{0}} \epsilon_{\mu 2} \partial_{\mu} \\
0 & -\partial^{2} & 0 \\
-2 h_{\mathrm{L}} \sqrt{1+\tilde{g}_{0}} \epsilon_{\mu 2} \partial_{\mu} & 0 & -\partial^{2}
\end{array}\right)
$$

and $m=(\vartheta, \varphi, \chi)$. $\left(\tilde{\lambda}_{0}, \tilde{g}_{0}\right.$ denote the bare coupling and parameter of the axion model). In Eq. (3.40) the $\epsilon$ tensor has been explicitly kept, as it requires a careful definition in $n=2-\epsilon$ dimensions used to regularize the momentum integrals. It is convenient to adopt the definition of Ref. [10], where this antisymmetric tensor corresponds to an almost complex structure: $\epsilon_{\mu \nu}=-\epsilon_{\nu \mu}, \epsilon_{\mu \nu} \epsilon_{\mu \sigma}=\delta_{\nu \sigma}$. The one loop quantum corrections to the classical ground state (the first term in 
Eq. (3.39)) require the calculation of a functional determinant, leading to

$$
\mathcal{F}(h)=\frac{4 h_{\mathrm{L}}^{2}}{n} \int \frac{d^{n} p}{(2 \pi)^{n}} \frac{\tilde{p}_{1}^{2}-\tilde{p}_{0} \tilde{p}_{2}^{2}}{\tilde{p}^{4}+4 h_{\mathrm{L}}^{2}\left(\tilde{p}_{1}^{2}-\tilde{g}_{0} \tilde{p}_{2}^{2}\right)}, \quad \tilde{p}_{\mu}=\epsilon_{\mu \nu} p_{\nu} .
$$

To evaluate (3.41) one should apply the modified dimensional regularization of Ref. [9] as $\tilde{p}_{2}=\epsilon_{2 \nu} p_{\nu}$ plays a distinguished rôle and it is kept as a one dimensional variable. In fact for our purposes it is sufficient to calculate the difference $\mathcal{F}(h)-$ $\mathcal{F}_{\Sigma}(h)$, where $\mathcal{F}_{\Sigma}(h)$ is the corresponding determinant in the anisotropic $\sigma$-model (Eq. (3.12) in Ref. [9]). Since both $\mathcal{F}(h)$ and $\mathcal{F}_{\Sigma}(h)$ are already the first quantum corrections to the classical expressions one may set $\tilde{g}=g$ (and make no distinction between bare and renormalized $g$ 's) when computing their difference to lowest order and one ends up with

$$
\mathcal{F}(h)-\mathcal{F}_{\Sigma}(h)=\frac{\left(2 h_{\mathrm{L}}\right)^{n}}{n}(1+g) \int \frac{d^{n} q}{(2 \pi)^{n}} \frac{\left(q_{1}^{2}-q_{2}^{2}\right) q^{4}}{N_{1} N_{2}}=\frac{\left(2 h_{\mathrm{L}}\right)^{n}}{n}(1+g) w(g),
$$

where $N_{1}=q^{4}+q_{1}^{2}-g q_{2}^{2}, N_{2}=q^{4}+q_{2}^{2}-g q_{1}^{2}$. Although the integrand yielding $w(g)$ is antisymmetric under $q_{1} \leftrightarrow q_{2}$, the integral is divergent by power counting for $n=2$, i.e. it must be computed in $n=2-\epsilon$ dimensions. Its derivative, $w^{\prime}(g)$, is, however, convergent by power counting and it has also an antisymmetric integrand, therefore this latter may be evaluated in $n=2$ dimensions giving $w^{\prime}(g) \equiv 0$. Then to compute $w(g)$ one may choose e.g. the point $g=-1$ :

$$
w(-1)=\int \frac{d^{n} q}{(2 \pi)^{n}} \frac{q_{1}^{2}-q_{2}^{2}}{\left(q^{2}+1\right)^{2}}=\frac{n-1-1}{n} \int \frac{d^{n} q}{(2 \pi)^{n}} \frac{q^{2}}{\left(q^{2}+1\right)^{2}}=-\frac{1}{4 \pi},
$$

where writing the second equality, it has been used that $q_{1}$ is an $n$-1-dimensional variable, while $q_{2}$ is 1-dimensional. From (3.43) one finds that after taking into account the change of the renormalization scheme (3.16), in PT the free energy densities of the two models (11.1) and (2.6) do indeed coincide for the BOS case.

I omit the details of the calculations in the FER case (see Ref. [11] for details), suffice it to say that this free energy is also in perfect agreement with the corresponding result in the anisotropic PCM model.

I would like to point out here that the canonical transformation connecting $\mathcal{L}_{\Sigma}$ and $\mathcal{L}$, maps $Q_{\mathrm{R}}$ to a purely topological charge $\tilde{Q}_{\mathrm{R}}$ of the axion model, which is completely different from the Noether charge of the 'manifest' $\mathrm{SO}(2)_{\theta}$ symmetry of the Lagrangian (1.1). To illustrate this I quote the value of the classical free energy density corresponding to the Noether charge of the $\mathrm{SO}(2)_{\theta}$ symmetry: $\left.\hat{H}\right|_{\min } ^{(\theta)}=-2 h_{\mathrm{R}}^{2} / \tilde{\lambda}$, very different indeed from Eq. (3.36) with $h_{\mathrm{L}}=0$.

In conclusion the free energy densities fully agree for the BOS and FER cases in both models up to first order in RG improved PT, providing a rather convincing evidence for the validity of T-duality at the quantum level. 


\section{Quantum integrability}

It is by now generally accepted that the anisotropic PCM model (2.6) is integrable at the quantum level [5, 9, [12] in the sense that there is no particle production. Assuming that the spectrum contains two massive doublets (kinks) their scattering is described by the tensor product of an $\mathrm{SU}(2) \times \mathrm{U}(1)$ symmetric solution of the bootstrap $S$-matrix equations:

$$
S(\theta)=S^{(\infty)}(\theta) \otimes S^{(p)}(\theta),
$$

where $S^{(p)}(\theta)$ denotes the Sine-Gordon (SG) S-matrix. Let me point out here that denoting the parameter in the $S$ matrix in the same way as the RG invariant quantity in Eq. (3.9),$(p)$, is intentional. As will become clear from the results later on, one should actually identify them. (In fact this identification is seen to be consistent at least up to two loops in PT.)

In the limit $p \rightarrow \infty$ (corresponding to $\bar{g} \equiv-1$ ) the symmetry of the $S$-matrix (4.1) increases to $\mathrm{SU}(2) \times \mathrm{SU}(2)$. For the range of the parameter $0<p<1$ in addition to the kinks bound states (breathers) also appear in the spectrum, transforming as $3+1$ under $\mathrm{SU}(2)$.

In this section I shall explore some consequences of assuming the validity of the duality transformation at the full quantum level between the two theories. This immediately implies the absence of particle production in the axion model (1.1) too, and that its two particle $S$-matrix is given by Eq. 4.1). Note that the global symmetries of the axion model, $\mathrm{SO}(3) \times \mathrm{SO}(2)_{\theta}$, cannot be identified with those of the $S$ matrix (4.1). As it will be convincingly shown the U(1) symmetry of the $S$ matrix (4.1) corresponds to a hidden 'topological' U(1) of the axion model, the dual of the $\mathrm{U}(1)_{\mathrm{R}}$ Noether symmetry of the anisotropic PCM under the T-duality transformation.

The following (rather heuristic) consideration might be useful to give some insight into the connection between the $\mathrm{SG}$ and the axion model. Integrating out the $\mathrm{O}(3)$ fields, $n^{a}$, in some generating functional of the theory (1.1) one obtains a non-vanishing effective potential for the $\theta$ field. Since $\theta$ is $2 \pi$-periodic the effective potential must be also periodic. The effective theory of the $\theta$ field is therefore expected to show some features similar to the Sine-Gordon model, with a periodic potential and corresponding topological current $K_{\mu}=\epsilon_{\mu \nu} \partial^{\nu} \theta / 2 \pi$. (Note that $\theta$ being an angular variable makes it difficult to integrate it out in the functional integral in spite of $\mathcal{L}$ being only quadratic in $\theta$.)

\subsection{Particle production in the $\mathrm{O}(3)$ model with a $\theta$-term}

At first sight the proposed quantum integrability of the axion model seems to be rather questionable at least, as it is generally believed that the $\mathrm{O}(3) \sigma$-model with a constant $\theta$-term is not quantum integrable, except for the special value 
$\theta=\pi$ [13] (despite the fact that the $\theta$-term, being a total derivative, does not change the classical physics of the model).

In the framework of the form-factor bootstrap approach one can actually show that the $\theta$ term mediates particle production, indeed [11]. To illustrate this important fact, let us express the $2 \rightarrow 3$ particle production amplitude to lowest order in $\theta$ as:

$$
\begin{gathered}
\left\langle p, b ; p^{\prime}, b^{\prime} ; p^{\prime \prime}, b^{\prime \prime} \mid q, a ; q^{\prime}, a^{\prime}\right\rangle_{(\theta)}=(2 \pi)^{2} i \theta \delta^{(2)}\left(p+p^{\prime}+p^{\prime \prime}-q-q^{\prime}\right) \\
\cdot\left\langle p, b ; p^{\prime}, b^{\prime} ; p^{\prime \prime}, b^{\prime \prime}|T(0)| q, a ; q^{\prime}, a^{\prime}\right\rangle_{(0)}+\mathcal{O}\left(\theta^{2}\right),
\end{gathered}
$$

where in the first line the amplitude is in the $\mathrm{O}(3)$ model with a $\theta$-term, while in the second line the matrix element of the topological charge density operator $T$ is to be calculated in the original $\mathrm{O}(3) \sigma$-model (with $\theta=0$ ). In other words one computes the $2 \rightarrow 3$ particle production amplitude to first order in $\theta$.

For the sake of simplicity let us consider the following special kinematical configuration: the incoming particles have momenta $q_{1}=Q$ and $q_{1}^{\prime}=-Q$, whereas the produced (outgoing) three particles have momenta $p_{1}=Q^{\prime}, p_{1}^{\prime}=0$ and $p_{1}^{\prime \prime}=-Q^{\prime}$ respectively. Here $Q^{\prime}$ can easily be expressed in terms of $Q$ and the kink mass $M$ using energy conservation. For large $Q$, using the results of Ref. [14], one finds

$$
\left\langle p, b ; p^{\prime}, b^{\prime} ; p^{\prime \prime}, b^{\prime \prime}|T(0)| q, a ; q^{\prime}, a^{\prime}\right\rangle_{(0)} \approx \pi^{\frac{5}{2}} \frac{Q^{2}}{\ln ^{3} Q / M}\left(\epsilon^{a^{\prime} b a} \delta^{b^{\prime \prime} b^{\prime \prime}}-\epsilon^{b^{\prime \prime} b a} \delta^{b^{\prime} a^{\prime}}\right) .
$$

Eq. (4.3) shows that already to first order in $\theta$, the $2 \rightarrow 3$ particle production amplitude is different from zero. Thus at least for small values of $\theta$, the introduction of this term destroys quantum integrability of the $\mathrm{O}(3) \sigma$-model, indeed. To the best of my knowledge this is the first quantitative evidence for the quantum non-integrability of the $\mathrm{O}(3) \sigma$-model with a constant $\theta$-term.

\subsection{Free energy from the Thermodynamical Bethe Ansatz}

The free energy of a system of particles of mass $m$ is of the form:

$$
\mathcal{F}(h)=-h^{2} F_{0}(h / m, Q) .
$$

The Thermodynamical Bethe Ansatz (TBA) method based on the $S$-matrix of the particles leads to a set of integral equations for the free energy whose solution can be found in many cases as an asymptotic series expansion in $h / m \gg 1$. Since $\mathcal{F}$ is a physical quantity, the results obtained by TBA method and by perturbation theory should agree. Thus by comparing the asymptotic series of $F_{0}(h / m, Q)$ in $h / m$ with the one of $F_{0}(h / \Lambda, Q)$ in $h / \Lambda$ obtained in PT provides a rather stringent consistency check on the $S$-matrix (and also on the self-consistency of the hypothesis used in the course of the calculation). Moreover one obtains the 
exact $m / \Lambda$ ratio, a rather important non-perturbative parameter of the theory which can be measured e.g. by lattice simulations.

For definiteness we choose $h_{\mathrm{L}}, h_{\mathrm{R}} \geq 0$ and assume $p>1$, implying that there are two doublets in the spectrum. The charges of the particles are normalized as:

$$
(1,1), \quad(-1,1), \quad(1,-1) \quad(-1,-1) .
$$

One can distinguish between the three possible types of finite density ground states depending on $h_{\mathrm{L}}, h_{\mathrm{R}}$ as:

1. $h_{\mathrm{L}}, h_{\mathrm{R}}>0$,

2. $h_{\mathrm{L}}=0, h_{\mathrm{R}}=h>0,(\mathrm{RIGHT})$

3. $h_{\mathrm{L}}=h>0, h_{\mathrm{R}}=0$. $(\mathrm{LEFT})$

- The DIAG case.

As the system (3.25) is considered with $h_{\mathrm{L}} / m, h_{\mathrm{R}} / m \gg 1$, it is clear that particles of charge $(1,1)$ condense into the vacuum. It is less clear what other kind of particles (necessarily with a smaller charge/mass ratio) will appear in the vacuum state. I shall assume that the vacuum consist of only particles of charge $(1,1)$. This seemingly radical assumption has apparently worked in all analogous examples studied so far, and it greatly simplifies the solution of TBA equations.

The scattering phase of the $(1,1)$ type particle, $\delta(\theta)$, can be easily found from the $S$-matrix:

$$
\delta(\theta)=\delta_{\infty}(\theta)+\delta_{p}(\theta) .
$$

When the ground state is assumed to contain only particles of charge $(1,1)$ the calculation of the free energy using the TBA method reduces to the solution of a single integral equation (4.8) whose kernel is given by the logarithmic derivative of the relevant $S$-matrix element,

$$
K(\theta)=\frac{1}{2 \pi i} \frac{d}{d \theta} \ln S(\theta) .
$$

The integral equation in this case is given as follows:

$$
\epsilon(\theta)-\int_{-B}^{B} d \theta^{\prime} K\left(\theta-\theta^{\prime}\right) \epsilon\left(\theta^{\prime}\right)=h-m \cosh \theta
$$

where $h=h_{\mathrm{L}}+h_{\mathrm{R}}$, together with the boundary condition $\epsilon( \pm B)=0$. In terms of the solution of Eq. (4.8) the free energy is

$$
\delta f(h)=-\frac{m}{2 \pi} \int_{-B}^{B} d \theta \cosh (\theta) \epsilon(\theta) .
$$

The asymptotic series for the free energy up to $O\left(\ln t / t^{3}\right)$ terms reads as:

$$
\delta f_{\mathrm{D}}(h)=-\frac{h^{2}}{\pi} p\left[1-\frac{p}{2 t}-\frac{p^{2}}{4} \frac{\ln t}{t^{2}}+\frac{A_{\mathrm{D}}}{t^{2}}+O\left(\frac{\ln t}{t^{3}}\right)\right],
$$


where $t=\ln (h / m)$ and $A_{\mathrm{D}}$ is a constant which determines the $m / \Lambda$ ratio:

$$
A_{\mathrm{D}}=\frac{p}{2}\left[\ln \Gamma\left(1+\frac{p}{2}\right)+\frac{p}{2}\left(\frac{3}{2}-\ln 2\right)-1+3 \ln 2-\ln \pi\right] .
$$

Let me remark here that from the classical term in Eq. (4.10), $-h^{2} p / \pi$, one can immediately read off the level of an underlying ultraviolet (UV) current algebra, $k$, as $k=p$ [15].

- The RIGHT case.

In analogy to the assumption made in the diagonal case one would now expect the ground state to consist of a mixture of particles of the same charge with respect to $\mathrm{U}(1)_{\mathrm{R}}$, i.e. those of charge $(1,1)$ and $(-1,1)$ with equal densities. In Ref. [12] the result of the diagonalisation of the pertinent coupled TBA system has been given and one finds (somewhat surprisingly) that

$$
\delta f_{\mathrm{R}}(h)=\delta f_{\mathrm{D}}(h),
$$

where $h=h_{\mathrm{R}}$. So the free energies are precisely the same as functions of the effective chemical potentials in these two apparently rather different cases.

- The LEFT case.

Analogously to the RIGHT case the ground state is expected to consist of an equal density mixture of particles of the same charge, this time with respect to $\mathrm{U}(1)_{\mathrm{L}}$, i.e. those of charge $(1,1)$ and $(1,-1)$. Here I just quote [9] the asymptotic series of the free energy density:

$$
\delta f_{\mathrm{L}}(h)=-\frac{h^{2}}{\pi}\left(t+\left(1-\frac{p}{2}\right) \ln t+A_{\mathrm{L}}+\ldots\right)
$$

where the constant $A_{\mathrm{L}}$, determining the ratio $m / \Lambda$ is:

$$
A_{\mathrm{L}}=\left(3-\frac{p}{2}\right) \ln 2+\frac{p-3}{2}-\ln \pi+\ln \Gamma\left(\frac{p}{2}\right) .
$$

- The LEFT case for $p<1$.

In the previous subsections it has been assumed that the parameter $p>1$. In order to clarify what happens when $p$ becomes smaller than one, I recall that then bound states (breathers) appear in the spectrum (for $p<1$ one enters simply the attractive regime). The SG mass spectrum is given by the well known formula:

$$
m_{r}=2 m \sin \frac{\pi p r}{2}, \quad r=1,2 \ldots<\frac{1}{p}
$$

where $m$ denotes the mass of the SG kinks. (Note that $m$ also gives the mass of the $\mathrm{SU}(2)$ doublets.) The appearance of these new particles implies that as soon as $p$ becomes smaller than 1 the vacuum changes as then it becomes energetically 
favorable for the $r=1$ charge $(2,0)$ breather to condense. This charge $(2,0)$ breather can be interpreted as the bound state of the $(1,1)$ and $(1,-1)$ particles (kinks). In fact as this particle has the highest charge/mass ratio, one can again assume (as in the DIAG case) that the true vacuum consists only of the condensate of the $r=1$ charge $(2,0)$ breather. Calculating the breather-breather phase shift by the bootstrap-fusion method [16] and solving the integral equation (4.8) yields the free energy density of the breather condensate, $\delta f_{\mathrm{B}}\left(h_{\mathrm{B}}, m_{1}\right)$, where $m_{1}=2 m \sin \frac{\pi p}{2}$ denotes the breather mass. One finds that $\delta f_{\mathrm{B}}\left(h_{\mathrm{B}}, m_{1}\right)$ becomes identical to the free energy of the charge $(1, \pm 1)$ kink mixture, $\delta f_{\mathrm{L}}(h, m)$ analytically continued for $p<1$. This is very reassuring as the result for $\delta f_{\mathrm{L}}(h, \Lambda)$ from perturbation theory turns out to be continuous for the full range $0 \leq p<\infty$, which is certainly to be expected.

\subsection{The $m / \Lambda_{\overline{\mathrm{MS}}}$ ratio}

Having calculated the asymptotic form of the free energy both in perturbation theory and with the TBA method, by comparing them (with $t=s-\ln \left(m / \Lambda_{\overline{\mathrm{MS}}}\right)$ ) one obtains the relation between the mass of the doublet particles, $m$, and $\Lambda_{\overline{\mathrm{MS}}}$. In both the BOS (LEFT) resp. FER (RIGHT,DIAG) cases the comparison of Eqs. (4.13) and (3.35) resp. (4.10) and (3.38) leads to the result

$$
\frac{m}{\Lambda_{\overline{\mathrm{MS}}}}=2^{3-\frac{p}{2}} e^{\frac{p}{2}-1} \frac{\Gamma(1+p / 2)}{\pi p} .
$$

I would like to emphasize that it is already a very nontrivial check on the overall consistency of the assumptions that all of the expansion coefficients, for both the BOS and the FER cases, agree.

To illustrate how nontrivial this consistency is, let me point out the following (apparent) paradox. In Eq. (4.10) the ratio of the coefficients of the $\ln t / t^{2}$ term, $-p^{2} / 4$, and the $-1 / t$ term, $p / 2$, is $r_{\mathrm{D}}=-p / 2$, while in Eq. (4.13) the ratio of the coefficients of the $\ln t$ term, $1-p / 2$, and that of the $t$ term is $r_{\mathrm{L}}=1-p / 2$. This is quite remarkable, since on very general grounds in a theory with a coupling, $\lambda$, the perturbative result for a fermionic type free energy is expected to be of the form

$$
\delta f_{\mathrm{Fer}}(h)=-\frac{h^{2} f_{0}}{\pi}\left(1-f_{1} \frac{\bar{\lambda}(h)}{2 \pi}+\mathcal{O}\left(\bar{\lambda}^{2}\right)\right),
$$

while in the same theory for a bosonic case it is expected to be given by

$$
\delta f_{\text {Bos }}(h)=-\frac{h^{2} b_{0}}{\pi}\left(\frac{1}{\bar{\lambda}(h)}+\text { const. }+\mathcal{O}(\bar{\lambda})\right),
$$

where $f_{0}, f_{1}, b_{0}$ are constants and $\bar{\lambda}(h)$ is the running coupling. Since from the renormalization group

$$
\frac{1}{\bar{\lambda}(h)}=\beta_{0} \ln \frac{h}{\Lambda}+\frac{\beta_{1}}{\beta_{0}} \ln \ln \frac{h}{\Lambda}+\mathcal{O}\left(\frac{1}{h / \Lambda}\right),
$$


one expects $r_{\mathrm{Fer}}=r_{\mathrm{Bos}}=\beta_{1} / \beta_{0}^{2}$. As in the present case $r_{\mathrm{D}} \neq r_{\mathrm{L}}$, it seems impossible to match Eq. (4.10) and Eq. (4.13) simultaneously with the corresponding perturbative expansion. The resolution of this paradox lies in the presence of the non-analytic contribution, $\ln \lambda_{\text {eff }}$, in the last term of Eq. (3.37). This non-analytic term in $\lambda_{\text {eff }}$ explains why the coefficient of the $\ln s$ term differs for the BOS and the FER cases. Such non-analytic terms can only occur in perturbation theory in the presence of several couplings.

A further, very stringent consistency check is that the $m / \Lambda$ ratio obtained in the BOS case is exactly the same as the one obtained in the FER case. Finally by comparing the corresponding free energies of the breather condensate one obtains the $m / \Lambda$ ratio in Eq. (4.16). This is clearly an additional check on our result and on the mutual consistency of the hypothesises made in the course of the calculation.

\subsection{The $p \rightarrow 0$ limit}

The $p \rightarrow 0$ limit (i.e. the running deformation parameter, $\bar{g}(h) \rightarrow-1$ ), is expected to give the $\mathrm{O}(3) \sigma$-model (and a decoupled free field) [17]. In fact from Eq. 4.15) one sees that $m / m_{1} \rightarrow \infty$ as $p \rightarrow 0$ i.e. the kinks 'disappear' from the spectrum. Furthermore the higher breathers with $r>1$ also 'disappear' from the spectrum, but for a different reason. The binding energy of the $r>1$ breathers (which can be considered as bound states of the $r=1$ ones) tends to zero giving rise to zero energy bound states. Therefore the limiting spectrum consists of 4 massive particles, transforming as $\left(3_{\mathrm{L}}+1_{\mathrm{L}}, 1_{\mathrm{R}}\right)$ under $\mathrm{SU}(2)_{\mathrm{L}} \times \mathrm{U}(1)_{\mathrm{R}}$. Our results lend some additional support to this expectation, as the $m_{1} / \Lambda_{\overline{\mathrm{MS}}}=8 / \mathrm{e}$ ratio in the $\mathrm{O}(3) \sigma$-model calculated previously [18] (where now $m_{1}$ stays for the mass of the triplet in the $\mathrm{O}(3)$ model) is beautifully reproduced in the $p \rightarrow 0$ limit. This can be immediately seen from Eq. (4.16) using the fact that in the $p \rightarrow 0$ limit $m_{1} \rightarrow \pi p m$

\section{Conclusions}

In conclusion one can say that there is complete consistency between the TBA and the perturbative calculations in both models, providing good evidence for the validity of the proposed $S$ matrix (4.1) for them. Since the effective coupling (3.11) is identical in the two models the $m / \Lambda_{\overline{\mathrm{MS}}}$ ratio Eq. (4.16) stays also the same. It might be worth emphasizing once again that these results provide also nonperturbative evidence for the validity of quantum T-duality. In particular one has some quantitative evidence for the identification between the $U(1)_{R}$ symmetry of the proposed $S$ matrix and the topological current of the axion model (corresponding to the canonical image of the $\mathrm{U}(1)_{\mathrm{R}}$ Noether current of the anisotropic PCM and being quite distinct from the manifest $\mathrm{SO}(2)_{\theta}$ symmetry). 
The above findings provide good motivation to study both models by lattice Monte-Carlo simulations. Measuring the $m / \Lambda_{L}$ ratios $\left(\Lambda_{L}\right.$ denoting the $\Lambda$ parameter of the lattice regularized theory) would provide us with a completely non-perturbative way of testing quantum T-duality.

\section{Acknowledgements}

I would like to thank the organizers of the Johns Hopkins Workshop 2000, in

particular Zalán and Laci, for providing me the opportunity to present the above results and Max Niedermaier for a careful reading of the manuscript.

\section{References}

[1] J. Balog, P. Forgács, Z. Horváth and L. Palla, Phys. Lett. 324B (1994) 403.

[2] T.H. Buscher, Phys. Lett. B201 (1988) 466, ibid. B194 (1987) 59.

[3] E. Alvarez, L. Alvarez-Gaumé, Y. Lozano, Phys. Lett. B336 (1994) 183.

[4] I.V. Cherednik, Theor. Math. Phys. 47 (1981) 422.

[5] A. Kirillov, N.Yu. Reshetikhin in Proc. of Proceedings of the Paris-Meudon Colloquium String Theory, etc. (1986), eds. N. Sanchez, H. de Vega, (World Scientific, Singapure).

[6] J. Balog, P. Forgács, Z. Horváth, L. Palla, Nucl. Phys. B (Proc. Suppl.) 49 (1996) 16. (hep-th/9601091)

[7] E. Alvarez, L. Alvarez-Gaumé, J.L.F. Barbón and Y. Lozano, Nucl. Phys. B415 (1994) 71.

[8] A.G. Bytsko, J. Math. Sciences 85 (1997) 1619. (hep-th/9403101)

[9] J. Balog and P. Forgács, Nucl. Phys. B570 (2000) 655.

[10] H. Osborn, Ann. Phys. 200 (1990) 1.

[11] J. Balog, P. Forgács, L. Palla, Phys. Lett. B484 (2000) 367

[12] V.A. Fateev, Nucl. Phys. B473[FS] (1996) 509.

[13] I. Affleck Field theory methods and critical phenomena, in Fields, strings and critical phenomena, ed. E. Brézin and J. Zinn-Justin (North Holland, Amsterdam,1990);

V.A. Fateev and Al.B. Zamolodchikov, Phys. Lett. 271B (1991) 91;

A.B. Zamolodchikov and Al.B. Zamolodchikov, Nucl. Phys. B379 (1992) 602.

[14] J. Balog and M. Niedermaier, Nucl. Phys. B500 (1997) 421.

[15] P. Fendley and K. Intriligator, Phys. Lett. 319B (1993) 132;

[16] M. Karowski, Nucl. Phys. B153 (1979) 244. 
[17] P.B. Wiegmann, Phys. Lett. 152B (1985) 209.

[18] A. Polyakov, P.B. Wiegmann, Phys. Lett. 131B (1984) 121, G. Japaridze, A. Nersesyan and P. Wiegmann, Nucl. Phys. B230 (1984) 511, P. Hasenfratz, M. Maggiore and F. Niedermayer, Phys. Lett. 245B (1990) 522. 